\title{
Editorial
}

\section{Problems with the continued proliferation of unsubstantiated psychophysiological techniques being promulgated and sold for clinical use}

Jerry R. DeVore \& Richard A. Sherman

Saybrook University, California-United States

OPEN

ACCESS

This journal is dedicated to publishing studies and reviews which assist our readers to recognize psychophysiological techniques, assessments, and interventions likely to be useful in a wide range of situations. Sadly, this includes informing readers when psychophysiological techniques are being promulgated and sold without sufficient evidence to support their claims of efficacy. This is crucially important because it is all too easy for most of us to mistake sales pitches supposedly supported by poor and nonexistent research, testimonials, and the like for actual evidence of efficacy. Thus, readers can't easily determine whether the technique in question has sufficient support to warrant its use. The journal will begin publishing thorough reviews of such techniques in the next few issues beginning with a review of low current and audiovisual stimulation techniques. An example of a technique which may well be effective but is being promulgated with claims far beyond the research demonstrating its efficacy is the LENS system currently being sold to treat a wide variety of clinical problems.

The LENS (Low-Energy Neurofeedback System) system is advertised as a neurofeedback system in which electroencephalographic (EEG) brain waves are assessed for the dominant frequency and then a low intensity (.0006 microamp) transcranial electric current (TACS) is administered for a few seconds. This brief, very low-intensity signal is said to reorganize cortical functioning and foster adaptive change for a wide variety of clinical conditions.

The current TACS system was preceded by a system developed by the same person named the Flexyx Neurotherapy System (FNS) that came on the market in the early 1990s. That system also assessed EEG waves for a few seconds, noted the dominant frequency, and generated a photic flicker at an offset to the dominant frequency in order to facilitate cortical dysregulation and reintegration. It was evaluated through several case studies (Shoenberger, et al, 2001) and one longer case series of fibromyalgia cases (Mueller, H.H., Donaldson, Stuart, 2001) which reported favorable results but there was no placebo control. Shortly after a randomized control / experimental group design was conducted (Kravitz, et al, 2001) with negative results, the system became unavailable.

Its successor is the LENS system which uses TACS. It began being broadly advertised in the early 2000s. It is supposed to rapidly help people with a variety of clinical conditions. According to the developer, he originally didn't realize that the earlier system (Flexyx) was sending a tiny electrical signal into the brain until he had engineers check the system as the results were far quicker than anticipated. 
It is this signal which he ascribes to be the means that produces effects so the Flexyx was abandoned and the LENS was developed.

Note that this is the traditional change when an unsubstantiated system is tested and found useless. You can read about hundreds of similar devices (e.g. the use of thermography for detection of breast cancer). As soon as the system is proven useless, the proponents make a minor change in the system and return to the market with specious claims unsupported by any controlled studies. Also, note that the proponents of such devices usually claim that appropriate studies are unnecessary as "you can see the changes" or that there is no adequate placebo for the device. Usually, but not always, the proponents encourage the use of the device by unlicensed or inappropriately licensed "practitioners" to avoid interference by the government. (An inappropriately licensed practitioner is one whose license does not include the use of such devices within the practitioner's scope of practice.) They also frequently ascribe the failure of the medical community to accept the device as being due to undue influence by various jealous powers. It is rare for such devices to have FDA (US Food and Drug Administration) labels indicating safety and efficacy. The few which do have them for some attribute of the device unrelated to the use promulgated for them (such as being used as transcutaneous electrical stimulation (TENS) units).

Readers can watch a 2011 discussion and demonstration of the photic system. A second video made in 2018 discusses case examples, the reasons why placebos are unnecessary as changes can observed happening, emphasizes patient testimonials, and briefly describes skin temperature monitoring at the millisecond level.

Data supporting the use of the LENS is extremely limited. The total amount of "research" on LENS appears to consist of (1) a book with uncontrolled case reports (Larsen, Stephen, 2006), The Healing Power of Neurofeedback: The Revolutionary LENS Technique for Restoring Optimal Brain Function, (2) the 2018 YouTube video noted above in which case reports are described, (3) Cripe, Curtis T, (2006) reported favorable outcomes on 4 individuals described as having neurodevelopmental trauma (note that this is not a specific diagnosis), (4) Hammond, Corydon, (2007) reported successful treatment in one case of anosmia resulting from TBI, (5) Hammond, Corydon (2010) reported on 2 cases on anger management, and (6) one case series of 100 participants (Larsen, Stephen, Harrington, Kristen and Hicks, Susan, 2006) with no comparable control group that reports favorable results following a series of LENS treatments.

There does not appear to be any published literature documenting any demonstrable effect, health-related or otherwise of a 1-2 second exposure of low-intensity (.0006microamps) TACS on cortical functioning. There are a number of entirely unsupported speculative hypotheses intended to explain the effect but there is no evidence that any of those hypotheses have been empirically examined.

It is crucial to remember that devices promulgated without placebo-controlled studies having sufficient subjects to demonstrate adequate power and sufficiently long follow-ups to show that initial effects last at least a few months are among those which usually disappear when adequately tested. There are thousands of these devices and techniques scattered across recent practice which disappear when adequately tested or simply hang on indefinitely. Due to the lack of adequate research, there is no way to tell whether LENS will join this sad group or go on to shine when appropriate studies are performed.

\section{References}

1. Cripe CT. Effective use of LENS unit as an adjunct to cognitive neuro- 
developmental training. J. Neurother. 2006; 10(2-3):79-87.

2. Hammond DC. Can LENS neurofeedback treat anosmia resulting from a head injury? Journal of Neurotherapy. 2007 Jun 20;11(1):57-62.

3. Hammond DC. Lens neurofeedback treatment of anger: Preliminary reports. J. Neurother. 2010; 14(2):162-169.

4. Kravitz HM, Esty ML, Katz RS, Fawcett J. Treatment of fibromyalgia syndrome using low-intensity neurofeedback with the Flexyx Neurotherapy System: A randomized controlled clinical trial. J. Neurother. 2006;10(2-3):41-58.

5. Larsen S, Harrington K, Hicks S. The LENS (low energy neurofeedback system): A clinical outcomes study on one hundred patients at Stone Mountain Center, New York. J. Neurother. 2006; 10(2-3):69-78.

6. Mueller HH, Donaldson CS, Nelson DV, Layman M. Treatment of fibromyalgia incorporating EEG-driven stimulation: a clinical outcomes study. J. Clin. Psychol. 2001; 57(7):933-952.

7. Schoenberger NE, Shiflett SC, Esty ML, Ochs L, Matheis RJ. Flexyx neurotherapy system in the treatment of traumatic brain injury: An initial evaluation. The JHRHE. 2001; 16(3):260-274.

8. Ochs L. Youtube. EEG driven light and sound stimulation at the Neurotechnology Forum (Seattle 1992). 2011. Available at: https://www.youtube.com/watch?v=h YOuX_kSfXo

9. Ochs L. Youtube. Mind Mending With Low Energy Neuro Feedback System. November 2018. Available at: https://www.youtube.com/watch?v=F z08GIgY_tA 